\title{
Viral macrophage inflammatory protein-II improves acute rejection in allogeneic rat kidney transplants
}

\author{
Jens Bedke • Tomislav Stojanovic • Eva Kiss • \\ Carl-Ludwig Behnes • Amanda E. Proudfoot • \\ Hermann-Josef Gröne
}

Received: 18 October 2009/ Accepted: 7 April 2010/Published online: 18 April 2010

(C) The Author(s) 2010. This article is published with open access at Springerlink.com

\begin{abstract}
Purpose During rejection, leukocytes are recruited from the peripheral circulation into the graft leading to the damage of endothelial cells, capillary perfusion failure and graft loss. Chemokines play a pivotal role in the recruitment of leukocytes to the endothelium. Viral macrophage inflammatory protein-II (vMIP-II), a human herpes virus-8 DNA-encoded protein, is a broad-spectrum chemokine antagonist. The aim of the study was to prove the beneficial activity of vMIP-II treatment on acute rat kidney allograft damage.

Methods Heterotopic rat kidney transplantation was performed in the Fischer 344 to Lewis transplantation model and animals were treated with vMIP-II $(2 \times 15 \mu \mathrm{g}$ or $100 \mu \mathrm{g} / \mathrm{day})$ for 7 days. Rejection-induced damage was analyzed by histology, and microcirculatory changes within the graft were analyzed by in vivo microscopy.
\end{abstract}

J. Bedke and T. Stojanovic contributed equally to this work.

Present Address:

J. Bedke $(\square)$

Department of Urology, Eberhard Karls University Tuebingen,

Hoppe-Seyler-Str. 3, 72076 Tübingen, Germany

e-mail: bedke@live.com

T. Stojanovic

Department of Thoracic, Heart and Vascular Surgery,

University of Göttingen, Göttingen, Germany

J. Bedke · E. Kiss · C.-L. Behnes · H.-J. Gröne

Department of Cellular and Molecular Pathology,

German Cancer Research Center, Heidelberg, Germany

A. E. Proudfoot

Merck Serono Pharmaceutical Research Institute,

Geneva, Switzerland
Results Viral macrophage inflammatory protein-II significantly improved acute glomerular damage and tubulointerstitial inflammation and lowered the extent of vascular and tubulointerstitial damage of the treated allografts. Functional microcirculation of peritubular capillaries was significantly improved in vivo, and the firm adherence of leukocytes was significantly reduced by vMIP-II treatment. Conclusions The administration of the broad-spectrum antagonist vMIP-II improved acute renal allograft damage, mainly by a reduction in leukocyte recruitment with a subsequently improved renal cortical microcirculation in vivo.

Keywords Chemokine - Virokine - vMIP-II · Inflammation · Allograft rejection

\section{Introduction}

The infiltration of leukocytes is a key feature in acute allograft rejection [1]. Endothelial cells play an important role in the rejection process because they promote both the recruitment and extravasation of recipient leukocytes [2]. On the endothelial surface glycosaminoglycans bind an immobilized gradient of chemokines, which upregulate integrin activity and thereby mediate the arrest of rolling leukocytes with the attraction of subgroups of leukocytes [3].

Chemokines are structurally related, low molecular weight $(8-12 \mathrm{kDa})$ proteins, which belong to a superfamily of molecules. They are divided into four groups based on the spacing of the first two conserved cysteines at the amino termini of the chemotactic proteins: XC, CC, CXC and $\mathrm{CX} 3 \mathrm{C}$ [3]. Chemokines have the ability to bind to multiple receptors and exert pleiotropic functions due to their redundant binding ability. This makes the chemokine 
network very robust, because blocking one specific chemokine receptor can be potentially overruled by the activation of other chemokine receptors. In acute and chronic renal allograft rejection, many chemokines and chemokine receptors can be detected in the graft, but relatively few appear to be of importance as demonstrated by animal studies. In acute renal allograft rejection, the selective blockade of the CC chemokine receptors CCR1, CCR5 and CXCR3 led to reduced allograft damage $[1,4,5]$.

Broad-spectrum chemokine antagonists may curtail the redundancy within the chemokine network. The Kaposi's sarcoma-associated human herpes virus- 8 encodes for the viral macrophage inhibitory protein II (vMIP-II). In vitro, this virokine has antagonistic activity on the chemokine receptors CCR1, CCR2, CCR5, CXCR3 and CXCR4, and is an agonist to CCR3 and CCR8. Furthermore, vMIP-II inhibits the chemotactic activity of the rat chemokines CCL2 (MCP-1), CCL4 (MIP-1 $\beta$ ), CCL5 (RANTES) on activated leukocytes [6]. In vivo vMIP-II prolongs allograft survival in a model of cardiac and corneal transplantation $[7,8]$.

The leukocyte-endothelial cell interaction has deleterious effects on perfusion of grafts peritubular capillaries. The deterioration of capillary perfusion can be measured prior to histological signs of acute rejection [9]. Thus, the graft's microcirculation is a sensitive parameter of rejection-induced damage [10]. Owing to the broadspectrum antagonistic activity, vMIP-II is an interesting candidate to ameliorate acute renal allograft rejection. The aim of this study was to test vMIP-II activity in acute rejection of renal allografts and to examine microvascular perfusion in peritubular capillaries in acute-rejecting rat kidneys by means of in vivo microscopy.

\section{Materials and methods}

Animals and renal transplantation experiment

Inbred male rats purchased from Charles River Laboratories GmbH (Sulzfeld, Germany) were used for all experiments. F344 RT1 ${ }^{\text {lvl }}$ rats $(230-250 \mathrm{~g})$ served as donors for kidney transplants. Lewis rats (LEW RT1 ${ }^{1}$, 200-230 g) served as recipients. All experiments were in accordance with the German legislation on the protection of animals. Renal transplantation was performed by a modified technique from Lee [11] as previously described [4]. The right kidney of the recipient was left in place to enhance allograft rejection [4] and to serve as a control for the effects of vMIP-II. Warm ischemia time of the donor kidney was $40 \mathrm{~min}$, cold ischemia time approximately $20 \mathrm{~min}$.
Experimental protocol and experimental groups

Viral macrophage inflammatory protein-II was kindly provided by Serono Pharmaceutical Research Institute (Geneva, Switzerland) and dissolved in $0.3 \mathrm{ml}$ sterile water at the respective dosage. The animals were treated with two different doses intravenously injected over the penile vein: A dose of $100 \mu \mathrm{g}$ once per day (HD-vMIP-II) or a dose of $15 \mu \mathrm{g}$ twice a day (LD-vMIP-II) was given. The initial dose of $100 \mu \mathrm{g} /$ day was chosen as previous experiments with the chemokine receptor antagonist Met-RANTES had proven to be beneficial in a rat kidney transplantation model [1] and then decreased to $30 \mu \mathrm{g}$ as the broad-spectrum antagonistic activity of vMIP-II might show inhibitory activity already at lower dosages.

The experimental groups were as follows:

Group 1, histology:

Group 1a: treated with sterile water for 7 days $(n=11)$.

Group $1 \mathrm{~b}$ : treated with vMIP-II at $2 \times 15 \mathrm{~g} /$ day for 7 days $(n=5)$.

Group 1c: treated with vMIP-II at $1 \times 100 \mu \mathrm{g} /$ day for 7 days $(n=4)$.

Group 2, in vivo microscopy:

Group 2a: treated with sterile water for 7 days $(n=8)$.

Group $2 \mathrm{~b}$ : treated with vMIP-II at $1 \times 100 \mu \mathrm{g} /$ day $(n=6)$.

Histological analysis

Renal allografts were removed in deep anesthesia, quickly blotted free of blood and processed as required for histology: The kidneys were cut into 2-mm slices and immersion fixed in $4 \%$ formaldehyde in phosphate buffered ( $\mathrm{pH} 7.35)$ saline (PBS, $99 \mathrm{mM} \mathrm{NaH} 2 \mathrm{PO} 4,108 \mathrm{mM}, \mathrm{NaH} 2 \mathrm{PO} 4$ and $248 \mathrm{mM} \mathrm{NaCl}$ ) for $24 \mathrm{~h}$ at $4{ }^{\circ} \mathrm{C}$ and then embedded in paraffin.

Renal morphologic studies

Light microscopy was performed on 3- $\mu$ m sections stained by periodic acid Schiff. In brief, the vascular injury of preglomerular vessels was assessed as showing no injury (0), sticking of mononuclear cells to the endothelium (0.5), subendothelial location of mononuclear cells (1), inflammation of the media, including transmural infiltration (without necrosis) (2) and fibrinoid necrosis of the vessel wall and thrombosis of the vessel in addition to the inflammatory reaction (3). The vascular injury was 
evaluated in whole kidney sections, including the cortex and outer stripe of outer medulla. An index specific vascular injury was defined as the percentage of vessels with the respective degree of injury encountered in a whole kidney section. The total vascular injury score was calculated as the sum of all specific vascular injuries, whereby the index of vessels with degree 0.5 was multiplied by 0.5 , that of degree 1 by a factor of 1 , that of degree 2 by a factor of 2 and that of degree 3 by a factor of 3 .

The glomerular, tubulointerstitial damage and tubulointerstitial inflammation scores were calculated in the same way as described for the vascular injury score [10].

\section{Intravital microscopy}

Intravital microscopy was performed as previously described and the technique was adopted for kidney analysis [9]. In brief, the abdomen was opened and the encapsulated kidney was placed on a specially constructed stage to avoid respiratory artefacts. Compression of the kidney did not occur. During microscopy, the kidney was continuously rinsed with warm $\left(37^{\circ} \mathrm{C}\right)$ Ringer solution. After a stabilization period of $15 \mathrm{~min}$, intraarterial injection of $0.6 \mathrm{ml} 0.5 \%$ fluorescein isothiocyanate-labeled dextran $(500,000 \mathrm{D})$ and $0.2 \mathrm{ml} \mathrm{0.1 \%}$ rhodamine-6G (both Sigma-Aldrich, Deisenhofen, Germany) were used to visualize renal cortical microcirculation and circulating leukocytes, respectively. Anaphylactic reactions (hypotension and limb edema) of the dyes were not observed.

By epi-illumination, a magnification of $\times 200$ and $\times 400$ was achieved with $\times 20$ and $\times 40$ water immersion objectives. At least ten sites of interest per animal were randomly selected and recorded by means of a CCD video camera (Kappa opto-electronics $\mathrm{GmbH}$, Gleichen, Germany).

Analysis of microcirculatory parameters in the renal cortex of the kidney was performed offline with an image analysis system (CapImage; Zeintl, Heidelberg, Germany). Functional capillary density was defined as the total length $(\mathrm{cm})$ of perfused capillaries per square centimeter. Red blood cell velocity was quantified with the line to shift diagram method in $\mathrm{mm} / \mathrm{s}[12,13]$.

Leukocyte endothelial interaction was analyzed as adherent leukocytes (stickers) in the peritubular capillary vessels and were defined as cells that did not move or detach from the vessel wall within an observation period of $30 \mathrm{~s}$. Leukocytes were counted as cells per observation area and expressed as cells per area $\left(\right.$ cells $\left./ \mathrm{mm}^{2}\right)$.

Statistical analysis

All data are presented as mean \pm standard error of mean (SEM). Data were analyzed by the two-sided non- parametric Mann-Whitney $U$ test; in case of multiple comparisons by Kruskal-Wallis test using GraphPad Prism (GraphPad, La Jolla, CA). A $p<0.05$ was considered to show a significant difference between two groups.

\section{Results}

Effects of vMIP-II on acute rejection and cell infiltration in allografts

In the untreated renal allografts, signs of tubulointerstitial rejection (interstitial infiltration and tubulitis), as well as a glomerular mononuclear cell infiltrate (transplant glomerulitis) and a vascular damage due to the subendothelial infiltration of mononuclear cells (endothelialitis, transmural arteritis) were observed by day 7 . Two concentrations of vMIP-II $(2 \times 15$ and $100 \mu \mathrm{g})$ were studied in the model. Both dosages of vMIP-II reduced glomerular injury (control vs. LD-vMIP-II vs. HD-vMIP-II; $33.0 \pm 8.0$ vs. $14.1 \pm 2.1$ vs. $8.8 \pm 3.0$ ), tubulointerstitial inflammation $(115.0 \pm 21.5$ vs. $67.0 \pm 16.2$ vs. $35.0 \pm 4.1)$ and tubular damage (11.0 \pm 3.8 vs. $8.5 \pm 2.7$ vs. $4.4 \pm 3.0)$ (Fig. 1a). Vascular cell infiltration was slightly improved (control vs. LD-vMIP-II vs. HD-vMIP-II; $45.23 \pm 7.5$ vs. $35.9 \pm 2.9$ vs. $33.1 \pm 6.0)$ (Fig. $1 \mathrm{a}-\mathrm{c})$. The high dose $(100 \mu \mathrm{g})$ had the most prominent effect with a significant reduction in tubulointerstitial inflammation (control vs. HD-vMIP-II, $p<0.05$ ) and glomerular injury (control vs. HD-vMIP-II, $p<0.05$ ) (Fig. 1a). The comparison between the high- and the low-dose treatment group did not show a significant difference between the groups, although a possible dosedependant effect was observed for the glomerular damage, tubulointerstitial inflammation and tubulointerstitial damage. Light microscopy showed no obvious effect of vMIP-II on the endogenous contralateral kidney (data not shown).

\section{Effect of vMIP-II on graft microcirculation in vivo}

Because tubulointerstitial inflammation was significantly reduced by day 7 in the HD-vMIP-II group, we performed an in vivo microscopy to determine microcirculatory changes of the allograft in vivo.

The untreated allografts showed a sludge of red blood and mononuclear cells within the capillaries which led to a capillary obstruction. The distribution of obstructed capillaries was discontinuous over the whole observation field with regularly perfused capillaries in between. The analysis of the functional capillary density, which was defined as the length of regularly perfused capillaries, was significantly higher in vMIP-II treated animals (control vs. HDvMIP-II, $99.2 \pm 31.4$ vs. $186.3 \pm 19.5, p<0.05$ ). Within the perfused capillaries, the red blood cell velocity did not 
Fig. 1 a Influence of vMIP-II treatment on acute rejectioninduced histologic changes: 7 days after transplantation, a reduction in acute rejection with lower glomerular damage, vascular rejection, tubulointerstitial inflammation and tubulointerstitial damage was observed in both dosages. The damage was significantly improved for the high dose treatment group as compared to control for glomerular damage and tubulointerstitial inflammation. Vascular rejection and tubulointerstitial damage were improved as compared to untreated controls, although not significantly. A dose-dependant effect on the reduction of acute rejectioninduced changes was noted for the glomerular damage, the tubulointerstitial inflammation and the tubulointerstitial damage (n.s. not significant, $p<0.05)$. b Untreated allografts show a high rate of adherent and transmigrated leukocytes at the vessel wall as a sign of acute vascular rejection in a preglomerular artery. $\mathbf{c}$ In contrast, vMIP-II treatment reduced signs of vascular rejection (endothelialitis), magnification $\times 400$
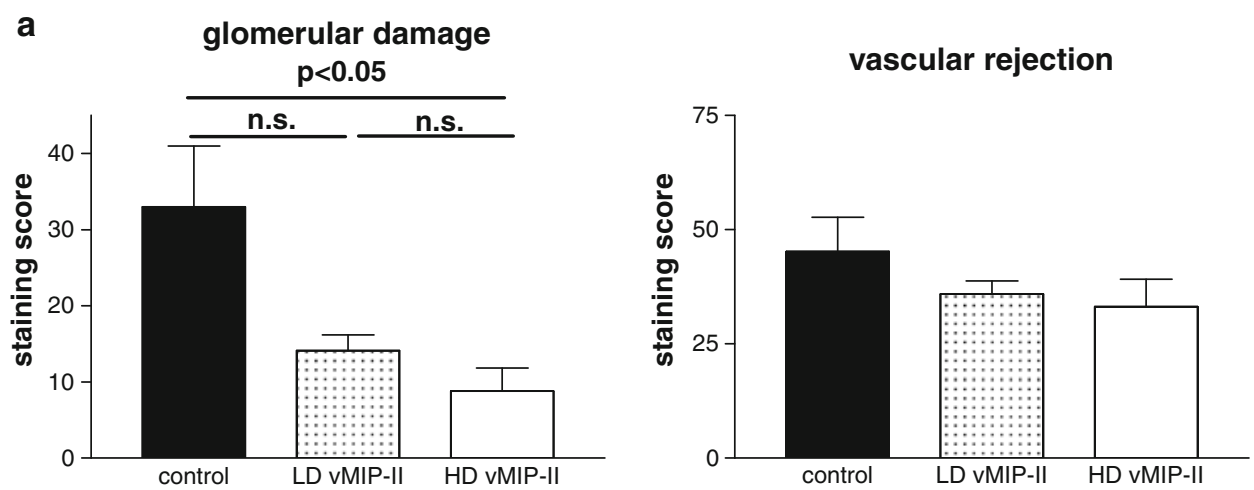

tubulointerstitial inflammation $p<0.05$

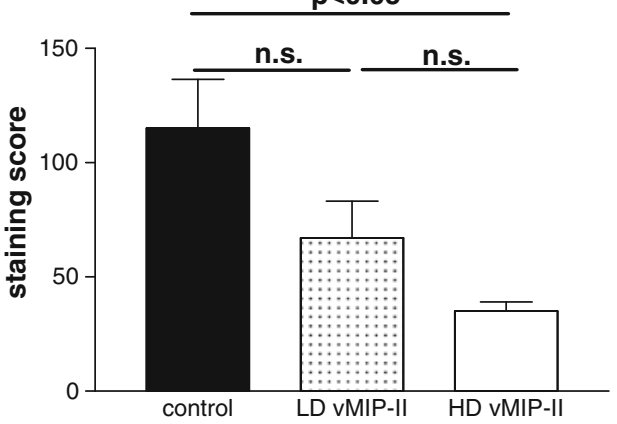

tubulointerstitial damage
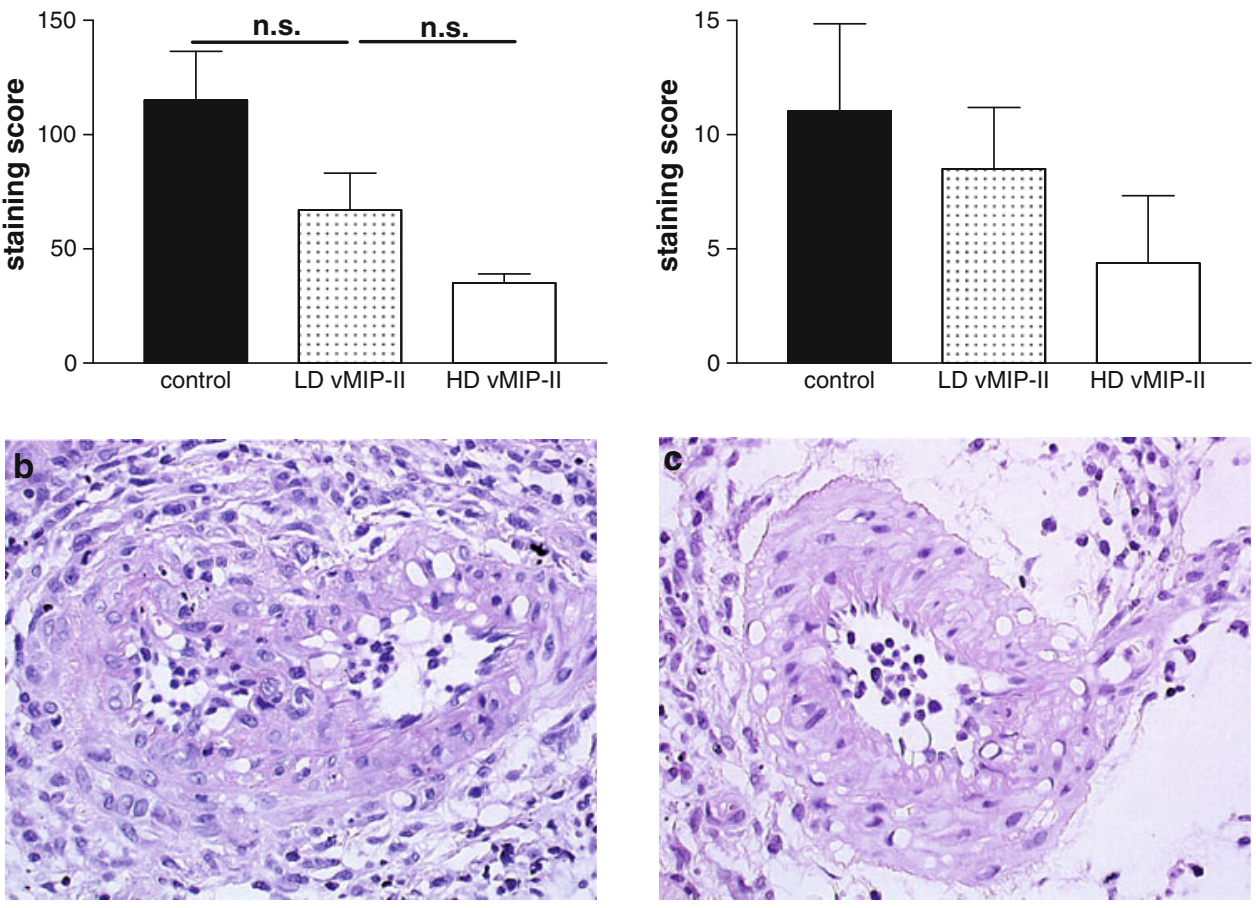

significantly differ between the groups (control vs. HDvMIP-II, $0.457 \pm 0.06$ vs. $0.534 \pm 0.06$ ) (Fig. 2).

Effect of vMIP-II on leukocyte adherence in vivo

Adherent leukocytes were prominent within the peritubular capillaries as seen by in vivo microscopy. Leukocytes tended to form clusters obstructing the capillaries. vMIP-II treatment significantly reduced leukocyte adherence in the grafts (control vs. HD-vMIP-II, $93.1 \pm 13.4$ vs. $61.2 \pm$ 7.4, $p<0.05$ ) (Fig. 2).

Mean arterial blood pressure measurements during in vivo microscopy did not reveal significant differences between the groups during the observation period (data not shown).

\section{Discussion}

The activation and transmigration of leukocytes from the peripheral capillaries into the interstitium of the graft is an important step in renal allograft rejection. The first step in leukocyte recruitment is the rolling of leukocytes along the endothelial surface through transient interactions between selectin molecules and their carbohydrate ligands. Then firm adherence to activated integrins is mediated by chemokines $[2,10]$. In this study, the broad-spectrum antagonist vMIP-II ameliorated acute rejection induced renal allograft damage; it significantly improved signs of acute glomerular damage and tubulointerstitial inflammation.

The significant reduction in mononuclear cells within the tubulointerstitium of vMIP-II-treated allografts 

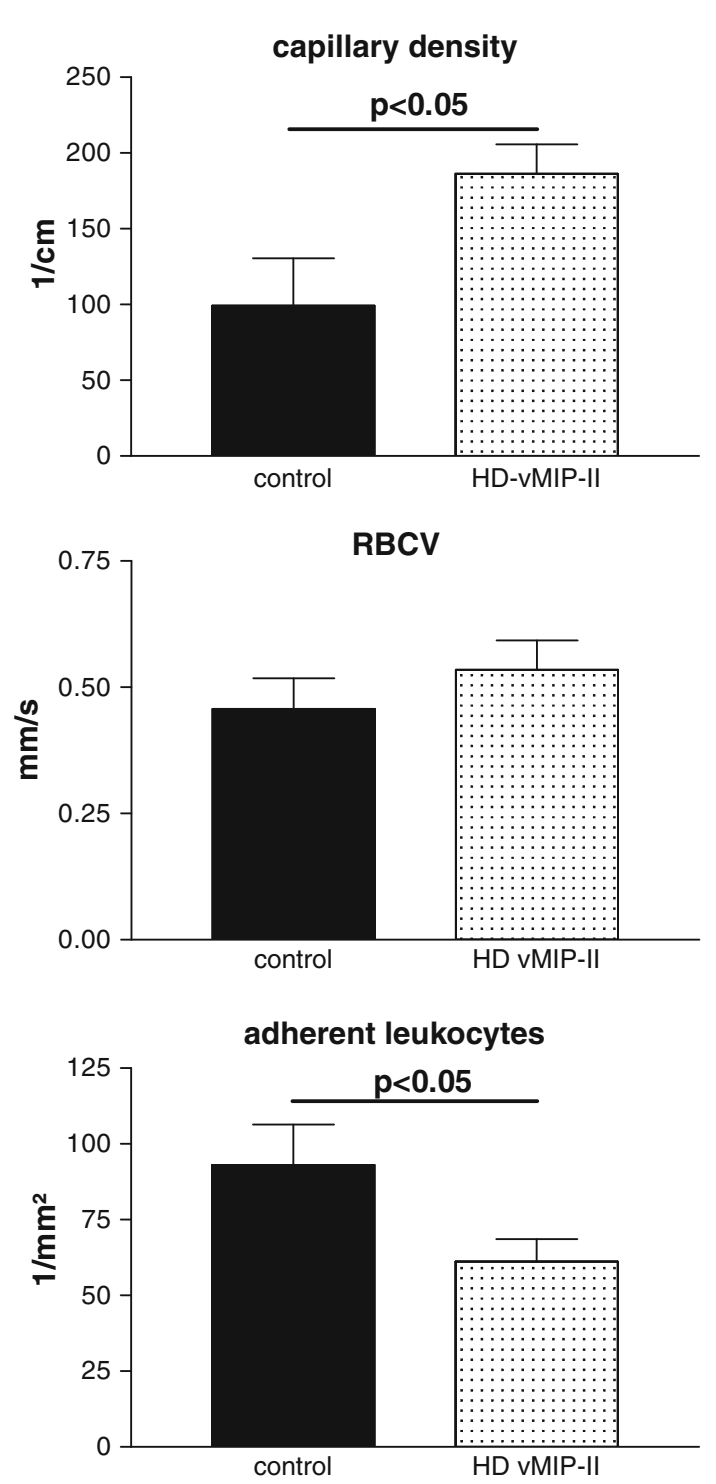

Fig. 2 Microcirculatory changes of vMIP-II treatment. vMIP-II treatment significantly improved functional capillary density $(p<0.05)$, while red blood cell velocity (RBCV) remained unchanged. Leukocyte-endothelial interaction was significantly reduced by vMIP-II showing fewer firm adherent leukocytes at the vessel wall in vivo $(p<0.05)$

prompted us to investigate microcirculatory changes and leukocyte-endothelial interaction in the peritubular capillaries of the allograft in vivo. In vivo microscopy is a sensitive method to detect microcirculatory changes in transplantation research even before histological signs of rejection become clearly visible $[9,14]$. In this study, we have demonstrated that vMIP-II administration improved functional capillary density, a sensitive marker of graft damage and reduced firm leukocyte adherence to the endothelium of peritubular capillaries in renal cortex.

By the method of in vivo microscopy, glomeruli could not be demonstrated due to the limited focus depth of the epi-illumination technique of approximately $50 \mu \mathrm{m}$. So far, direct analysis of flow changes within the afferent and efferent arterioles and of glomeruli can only be accomplished in congenital or experimentally induced hydronephrotic rats with superficial glomeruli [15].

A reduction in leukocytes firmly adhering to the vessel wall in vMIP-II-treated animals was also observed in an in vitro experiment, by which vMIP-II inhibited the RANTES-triggered arrest of monocytes and Th1-like lymphocytes to an endothelial layer [16]. Here, vMIP-II prevented CCR1 and CCR5 receptor internalization, which mediate arrest and transmigration of these cells [16].

Ischemia/reperfusion (I/R) injury at the time of transplantation is known to negatively influence later graft outcome. I/R injury is a risk factor for acute rejection. In a cerebral infarct model, the intracerebroventricular injection of vMIP-II in a mice model reduced infarct volume in a dose-dependant manner [17]. Although the doses of 0.01$1 \mu \mathrm{g}$ used in that model were much lower as the ones in our renal transplantation experiment, $\mathrm{I} / \mathrm{R}$ injury might have been beneficially influenced by vMIP-II treatment at the time of reperfusion of the allograft. The reduced I/R injury can subsequently reduce the degree of developing acute allograft rejection.

A Th1-immune response is believed to be important during acute renal transplant rejection [18]. Studies of chemokine expression during acute rejection showed an increased abundance of mRNAs encoding chemokines such as interferon-inducible protein-10 (IP-10), RANTES, MIP- $1 \alpha$, MIP- $1 \beta$ and lymphotactin, as well as the chemokine receptors CCR2 and CCR5, in the absence of upregulation of CCR3 and CCR8, which are Th2 chemokine receptor phenotypes [19]. vMIP-II inhibited the firm arrest and transmigration of Th1 cells under in vitro flow conditions and exerted an agonistic action on CCR3 driving a Th2 immune response [16]. Other groups reported that gene transfer of plasmid DNA encoding vMIP-II into murine cardiac allografts resulted in a prolonged survival time of allografts and a marked decrease in donor-specific cytotoxic T lymphocytes (CTL) infiltrating the grafts with an inhibition of alloantibody production [8]. vMIP-II administration has been reported to reduce leukocyte infiltration and glomerulitis in an experimental model of glomerulonephritis by reducing the chemotactic activity of MCP-1, MIP-1 $\beta$, RANTES and fractalkine-induced chemotaxis of activated leukocytes [6].

The redundancy of the chemokine network was effectively broken by the viral chemokine antagonist vMIP-II in this study. The result was a lower acute rejection-induced damage, which was to some extent still present. This shows that vMIP-II, as a peptide chemokine receptor antagonist, without a degradation inhibiting protection, is not potent enough to fully prevent acute rejection-induced damage 
and perfusion disturbance. Nevertheless, a trend to a dosedependant effect was observed. The extent of glomerular damage, tubulointerstitial inflammation and tubulointerstitial damage was additionally lowered by a high dose treatment.

A side effect of vMIP-II which dampens the antiinflammatory response is the concomitant agonistic activity on Th2-associated receptors, such as CCR3 provoking a Th2-type response [16]. Data about the antagonistic activity on CCR5 are contradictory, as vMIP-II as a chemoattractant attracted CCR5-positive monocytic THP 1 cell in vitro [20]. On the other hand, vMIP-II significantly reduced CCL5-induced rolling of Th1 cells in the ear vasculature of mice [21]. Here, specific chemokine receptor inhibitors might allow a more specific analysis to reduce acute renal allograft injury. The peptide MetRANTES inhibits CCR1 and CCR5 and is better protected to protein degradation by the added amino acid methionine. The small molecule antagonist BX471 inhibits specifically CCR1 and repertaxin inhibits specifically CXCR1/2. For example, the selective blockade of CCR1 not only ameliorated acute renal allograft damage, but also beneficially influenced chronic allograft nephropathy [1, 4, 22].

In conclusion, the administration of the broad chemokine analog vMIP-II improved the microvascular perfusion and the histological parameters associated with acute rejection. Blocking chemokine receptors and/or function during acute allograft rejection reduced the adherence of leukocytes to the endothelium of peritubular capillaries. These results encourage further investigation of chemokine receptor blockade by broad-spectrum antagonists during acute allograft rejection. This may further offer the possibility of a dose reduction in immunosuppressants and hence lower immunosuppressive-induced adverse effects.

Acknowledgments The authors like to thank Mrs. Claudia Schmid for expert technical assistance. The study was supported by a Grant given to H.-J. Gröne (SFB 405, B10).

Conflict of interest statement Amanda E Proud foot is an employee of Merck Serono Geneva Research Center, Merck Serono International.

Open Access This article is distributed under the terms of the Creative Commons Attribution Noncommercial License which permits any noncommercial use, distribution, and reproduction in any medium, provided the original author(s) and source are credited.

\section{References}

1. Grone HJ et al (1999) Met-RANTES reduces vascular and tubular damage during acute renal transplant rejection: blocking monocyte arrest and recruitment. FASEB J 13:1371-1383
2. Springer TA (1994) Traffic signals for lymphocyte recirculation and leukocyte emigration: the multistep paradigm. Cell 76:301314

3. Nelson PJ, Krensky AM (2001) Chemokines and allograft rejection: narrowing the list of suspects. Transplantation 72:1195-1197

4. Bedke J et al (2007) Beneficial effects of CCR1 blockade on the progression of chronic renal allograft damage. Am J Transplant 7:527-537

5. Hancock WW et al (2003) Chemokines and their receptors as markers of allograft rejection and targets for immunosuppression. Curr Opin Immunol 15:479-486

6. Chen S et al (1998) In vivo inhibition of CC and CX3C chemokine-induced leukocyte infiltration and attenuation of glomerulonephritis in Wistar-Kyoto (WKY) rats by vMIP-II. J Exp Med 188:193-198

7. Pillai RG et al (2008) Expression of the chemokine antagonist vMIP II using a non-viral vector can prolong corneal allograft survival. Transplantation 85:1640-1647

8. DeBruyne LA et al (2000) Gene transfer of virally encoded chemokine antagonists vMIP-II and MC148 prolongs cardiac allograft survival and inhibits donor-specific immunity. Gene Ther 7:575-582

9. Stojanovic T et al (2002) Met-RANTES inhibition of mucosal perfusion failure in acute intestinal transplant rejection-role of endothelial cell-leukocyte interaction. J Vasc Res 39:51-58

10. Bedke $\mathbf{J}$ et al (2007) Anti-inflammatory effects of alphav integrin antagonism in acute kidney allograft rejection. Am J Pathol 171:1127-1139

11. Lee S (1967) An improved technique of renal transplantation in the rat. Surgery $61: 771$

12. Klyscz T et al (1997) Cap image-a new kind of computerassisted video image analysis system for dynamic capillary microscopy. Biomed Tech (Berl) 42:168-175

13. De Vriese AS et al (2000) Off-line analysis of red blood cell velocity in renal arterioles. J Vasc Res 37:26-31

14. Menger MD, Vollmar B (2000) Role of microcirculation in transplantation. Microcirculation 7:291-306

15. Heuser $M$ et al (2001) Differences in cortical microcirculation in the kidneys of unilaterally congenital hydronephrotic rats. Microvasc Res 62:172-178

16. Weber KS et al (2001) Selective recruitment of Th2-type cells and evasion from a cytotoxic immune response mediated by viral macrophage inhibitory protein-II. Eur J Immunol 31:2458-2466

17. Takami S et al (2001) Chemokine receptor antagonist peptide, viral MIP-II, protects the brain against focal cerebral ischemia in mice. J Cereb Blood Flow Metab 21:1430-1435

18. Ode-Hakim S et al (1996) Delayed-type hypersensitivity-like mechanisms dominate late acute rejection episodes in renal allograft recipients. Transplantation 61:1233-1240

19. Segerer $\mathrm{S}$ et al (2001) Expression of chemokines and chemokine receptors during human renal transplant rejection. Am J Kidney Dis 37:518-531

20. Nakano K et al (2003) Kaposi's sarcoma-associated herpesvirus (KSHV)-encoded vMIP-I and vMIP-II induce signal transduction and chemotaxis in monocytic cells. Arch Virol 148:871-890

21. Rubant $S$ et al (2006) Eukaryotic expression of the broad-spectrum chemokine receptor antagonist vMIP-II and its effects on Tcell function in vitro and in vivo. Exp Dermatol 15:634-642

22. Cugini D et al (2005) Inhibition of the chemokine receptor CXCR2 prevents kidney graft function deterioration due to ischemia/reperfusion. Kidney Int 67:1753-1761 